\title{
New Horizons: Long-range Kuiper Belt targets observed by the Hubble Space Telescope
}

\author{
S.D. Benecchi ${ }^{\mathrm{a}, \mathrm{b}, *}$, K.S. Noll $^{\mathrm{c}}$, H.A. Weaver ${ }^{\mathrm{d}}$, J.R. Spencer ${ }^{\mathrm{e}}$, S.A. Stern ${ }^{\mathrm{e}}$, M.W. Buie ${ }^{\mathrm{e}}$, A.H. Parker ${ }^{\mathrm{f}}$ \\ a Planetary Science Institute, 1700 East Fort Lowell, Suite 106, Tucson, AZ 85719, United States \\ ${ }^{b}$ Carnegie Institution of Washington, Department of Terrestrial Magnetism, 5241 Broad Branch Road, NW, Washington, DC 20015, United States \\ ${ }^{\text {c } N A S A ~ G o d d a r d ~ S p a c e ~ F l i g h t ~ C e n t e r, ~} 8800$ Greenbelt Road, Code 693, Greenbelt, MD 20771, United States \\ d Space Department, Johns Hopkins University Applied Physics Laboratory, 11100 Johns Hopkins Road, Laurel, MD 20723, United States \\ e Southwest Research Institute, 1050 Walnut St., Suite 300, Boulder, CO 80302, United States \\ ${ }^{\mathrm{f}}$ Department of Astronomy, University of California at Berkeley, B-20 Hearst Field Annex \#3411, Berkeley, CA 94720, United States
}

\section{A R T I C L E I N F O}

\section{Article history:}

Received 5 February 2014

Revised 14 April 2014

Accepted 15 April 2014

Available online 2 May 2014

\section{Keywords:}

Kuiper Belt

Photometry

Hubble Space Telescope observations

\begin{abstract}
A B S T R A C T
We report on Hubble Space Telescope (HST) observations of three Kuiper Belt Objects (KBOs), discovered in our dedicated ground-based search campaign, that are candidates for long-range observations from the New Horizons spacecraft: $2011 \mathrm{JY}_{31}, 2011 \mathrm{HZ}_{102}$, and $2013 \mathrm{LU}_{35}$. Astrometry with HST enables both current and future critical accuracy improvements for orbit precision, required for possible New Horizons observations, beyond what can be obtained from the ground. Photometric colors of all three objects are red, typical of the Cold Classical dynamical population within which they reside; they are also the faintest KBOs to have had their colors measured. None are observed to be binary with HST above separations of $\sim 0.02 \operatorname{arcsec}(\sim 700 \mathrm{~km}$ at $44 \mathrm{AU})$ and $\Delta m \leqslant 0.5$.
\end{abstract}

(c) 2014 Elsevier Inc. All rights reserved.

\section{Introduction}

The New Horizons spacecraft, launched in January 2006, will fly through the Pluto system in July 2015. The Pluto system, consisting of a large binary $\left(d_{\text {Pluto }}=2306 \mathrm{~km}\right.$, and $\left.d_{\text {Charon }}=1307 \mathrm{~km}\right)$ with four smaller satellites $\left(d_{\text {satellites }} \sim 10-170 \mathrm{~km}\right)$, is one of the largest and most complex members of the Kuiper Belt (Jewitt and Luu, 1993). This region has more than 1700 objects that have been discovered and cataloged, and is estimated to contain $\sim 10^{5}$ objects having diameters larger than $d \sim 100 \mathrm{~km}$ (Petit et al., 2011). The physical properties of smaller $(d \sim 30-300 \mathrm{~km})$, less atypical Kuiper Belt Objects (KBOs) provide important points of comparison and context for understanding the Pluto system and its relationship to the larger Kuiper Belt. A flyby of a KBO and studies of other encounterable objects have always been key goals for the New Horizons mission beyond the Pluto encounter as identified for the NASA KBO/Pluto mission (which became New Horizons) in the 2002 Planetary Decadal Survey (Belton et al., 2002). The New Horizons spacecraft and mission was designed, therefore, to allow redirection to one or more KBOs between 2017 and 2021 (Stern, 2008; Spencer et al., 2003) provided that a suitable candidate can

\footnotetext{
* Corresponding author at: Planetary Science Institute, 1700 East Fort Lowell, Suite 106, Tucson, AZ 85719, United States.

E-mail address: susank@psi.edu (S.D. Benecchi).
}

be identified. This is likely to be the only opportunity in our lifetimes to encounter a KBO other than Pluto.

Because precise orbits for flyby and long-range candidates are critical to the success of the New Horizons extended mission, we present in this paper Hubble Space Telescope (HST) follow-up observations of candidate targets exploiting HST's sensitivity, resolution, and PSF stability. These HST observations also allow us to make basic color measurements and to search for binary companions down to $\sim 0.02$ arcsec in angular separation at the time of the observations.

\subsection{Identifying potentially encounterable KBOs}

The KBOs that are potentially encounterable by New Horizons are limited to those with orbits that place them within reach of the remaining $\Delta v$ capability of the spacecraft, currently estimated to be $\Delta v \approx 130 \mathrm{~m} \mathrm{~s}^{-1}$. This restriction means that by far the most likely candidate target(s) will be a member of the low-eccentricity, low-inclination Cold Classical subpopulation. In 2004 a groundbased search for bright objects $\left(m_{R} \geqslant 24.0\right)$ was conducted over a wide area of sky (a few degrees) through which objects that will be in the New Horizons spacecraft trajectory during the optimal KBO encounter dates were located. This is defined by projecting the nominal trajectory of New Horizons after the Pluto encounter on the sky, then tracking the orbits of those potential KBOs 
backward (and forward) in time and orbital space. The total sky area that these potential KBO orbits cover is larger the farther out in time from encounter one searches. The closer the search time is to encounter, the more collapsed the potential KBO orbits are in the sky search area, however, this also means a shorter astrometry baseline for accurate obit determination and targeting of the discovered KBO. Unfortunately, no bright accessible object was found in the initial survey (Buie et al., 2012). In 2011, when ground-based searching conditions became optimized - the search area was small enough to justify the use of significant amounts of observing time on large telescopes - a deep search campaign (ideally to $m_{R} \sim 26.5$, but more realistically to $m_{R} \sim 26.0$ ) was begun in earnest. As of January 2014, we have utilized over $520 \mathrm{~h}$ ( $\sim 52$ nights) of telescope time on large aperture facilities (the $8.4-\mathrm{m}$ Subaru telescope with SuprimeCam and the 6.5-m Magellan telescopes with the MegaCam and IMACS cameras; Buie et al., 2012; Spencer et al., 2013). No KBO has been found yet that is within the $\Delta v$ fly-by capabilities of New Horizons.

Identifying encounterable KBOs has been especially challenging because the search region is near the galactic plane where the high star density and consequent confusion limit the depth to which we can effectively search. Additionally, there appears to be a turnover in the KBO magnitude distribution at small (faint) sizes (Fraser et al., 2014) that is more severe than originally anticipated. It is desirable to detect potential encounter objects as early as possible because the uncertainty in the predicted location of the KBO encounter candidate depends critically on the of the orbital arc length of the object. Some of the phase space where KBO encounter objects could be found is lost if retargeting of the spacecraft cannot be done shortly after the Pluto system encounter. A minimum of a 1 -year arc on the KBO orbit is necessary for the initial burn, with continued orbit refinement closer to the time of the KBO fly-by maneuver itself.

However, a targeted close fly-by of an object is not the only New Horizons KBO science available. Longer range observations of objects, passing within $0.3 \mathrm{AU}$ of New Horizons that are bright enough to image from the spacecraft with $\sim 150 \mathrm{~km}$ resolution or $\sim 5 \times$ HST resolution, provide opportunities for measurements with the New Horizons instruments that exceed the capabilities of Earth-based facilities and that provide unique phase angle coverage not available for any objects observed from the Earth. At the time of this paper our team has discovered $52 \mathrm{KBOs}$ including a high inclination Neptune Trojan $2011 \mathrm{HM}_{102}$ (Parker et al., 2013a). At least five of these objects are long-range observation candidates $(<0.3 \mathrm{AU})$ with ground-based orbital arcs sufficient for HST targeting, including the four objects presented in this paper (Table 1). Follow-up observations of these objects were prioritized by their magnitudes, orbital arcs and an evaluation of their encounter probabilities based on Monte Carlo models of their projected orbits with respect to the New Horizons spacecraft trajectory and the $\Delta v$ available to the spacecraft for maneuvering. Four additional objects among our discoveries, but not presented here, were found to have preliminary predictions to pass within long range targeting by New Horizons, however, they were not recovered enough times in our ground-based data to extend their orbital arcs beyond a few nights to confirm their long range observation candidacy and to guarantee observation within the HST field of view. In most cases, their magnitudes were also significantly uncertain. Details of our ground-based campaign will be presented in a future paper.

\section{Observations}

Observations were obtained using the Wide Field Camera 3 (WFC3) in the UVIS channel on HST (0.04 arcsec/pixel, field of view 162 arcsec square). Objects were tracked at the rate of motion of the $\mathrm{KBO}$ and observations were made using the F606W filter, nominally centered at $600.1 \mathrm{~nm}$ with FWHM of $150.2 \mathrm{~nm}$, comparable to a Johnson V filter and the F814W filter, centered at $799.6 \mathrm{~nm}$ with FWHM $152.2 \mathrm{~nm}$, comparable to Cousins I (Heyer et al., 2004). The V-I colors (or the equivalent colors in HST filters) of the candidate objects can determine if they have the red colors characteristic of Cold Classical KBOs (Gulbis et al., 2006; Peixinho et al., 2008). If we found an object with a neutral or blue color, it might potentially be an interloper from another dynamical class of the Kuiper Belt. With multiple candidate targets for distant observations with New Horizons, objects with a spread of colors might provide the most useful scientific insight to the properties of the Kuiper Belt region if in fact their surfaces sampled different populations in the Kuiper Belt. In Cycle 19, September 2012, we observed three of the best long-range candidates (HST 12535: $2011 \mathrm{JW}_{31}, 2011 \mathrm{JY}_{31}$, and $2011 \mathrm{HZ}_{102}$ ) and in Cycle 20, September 2013, we observed the newly discovered long-range candidate 2013 LU $_{35}$ (HST 12887). For each object a pair of HST orbits were utilized, obtaining both astrometric and photometric measurements.

Observations for each orbit followed the sequence: F606WF814W-F814W-F606W with small dithers between each exposure and with 1-15 HST orbit separation, so that the KBOs could be seen to move relative to the background star field between orbits. Exposures were $455 \mathrm{~s}$ in F606W and $640 \mathrm{~s}$ in F814W with the goal of obtaining $\mathrm{S} / \mathrm{N} \sim 5$ for a single image, and color measurements good to 0.1 magnitudes with the combined images. Table 2 summarizes the raw observations. Because these objects are in high density star fields, HST observation times were pre-selected based on the best known star catalogs to limit the probability of being close to, or overlapping, bright stars in the field of view. A sample HST image for each object in a single F606W integration are given in Fig. 1.

\section{Detection, photometric and astrometric analysis}

\subsection{Detection and photometry}

To identify the KBOs of interest in our fields, the HST images were registered and then stacked based on the expected plane

Table 1

Long range candidates.

\begin{tabular}{|c|c|c|c|c|c|c|c|c|}
\hline Name & $a(\mathrm{AU})$ & $e$ & $i\left({ }^{\circ}\right)$ & Date of enc. & Range at enc. $(\mathrm{AU})^{\mathrm{c}}$ & Lorri resolution $(\mathrm{km})^{\mathrm{b}, \mathrm{c}}$ & $\Delta v$ Required for enc. $(\mathrm{m} / \mathrm{s})^{\mathrm{c}}$ & Obs arc (days) \\
\hline $2011 \mathrm{HZ}_{102}^{\mathrm{a}}$ & 43.2 & 0.008 & 2.4 & $2018 / 12 / 16-2018 / 12 / 16$ & $0.15-0.16$ & $111-119$ & $210-210-210$ & 801 \\
\hline $2011 \mathrm{JY}_{31}^{\mathrm{a}}$ & 44.0 & 0.057 & 2.6 & 2018/09/17-2018/09/17 & $0.16-0.16$ & 118 & $230-230-240$ & 801 \\
\hline $2011 \mathrm{JW}_{31}^{\mathrm{a}}$ & 45.6 & 0.094 & 1.9 & $2018 / 09 / 27-2018 / 09 / 27$ & $0.19-0.19$ & 141 & $280-280-280$ & 809 \\
\hline $2011 \mathrm{HJ}_{103}$ & 49.6 & 0.322 & 5.4 & $2017 / 02 / 24-2018 / 01 / 10$ & $0.19-0.43$ & $141-319$ & $530-730-880$ & 66 \\
\hline $2013 \mathrm{LU}_{35}{ }^{\mathrm{a}}$ & 43.8 & 0.024 & 3 & 2019/02/04-2019/10/12 & $0.30-0.38$ & $222-282$ & $360-430-450$ & 95 \\
\hline
\end{tabular}

a Designates an object we attempted to observe with HST

b 1 Lorri pixel = 5.959 microradians (Cheng et al., 2008).

c The first and last intervals are the 2.5 th percentile, 97.5 th percentile (the interval enclosing the central $95 \%$ of the posterior probability distribution) of 200 samples. The middle value in column 8 is the peak of the probability distribution (the modal velocity). 
Table 2

Observations.

\begin{tabular}{|c|c|c|c|c|c|c|}
\hline Object & Image & JD & Calendar date & Filter & Exptime (s) & Magnitude \\
\hline $2011 \mathrm{JW}_{31}$ & ibr001faq & 2456188.53241 & $2012 / 09 / 18.03241$ & F606W & 455 & $\mathrm{~N} / \mathrm{A}$ \\
\hline $2011 \mathrm{JW}_{31}$ & ibr001fbq & 2456188.54088 & $2012 / 09 / 18.04088$ & F814W & 640 & $\mathrm{~N} / \mathrm{A}$ \\
\hline $2011 \mathrm{JW}_{31}$ & ibr001feq & 2456188.55015 & $2012 / 09 / 18.05015$ & F814W & 640 & $\mathrm{~N} / \mathrm{A}$ \\
\hline $2011 \mathrm{JW}_{31}$ & ibr001g3q & 2456188.55862 & $2012 / 09 / 18.05862$ & F606W & 455 & $\mathrm{~N} / \mathrm{A}$ \\
\hline $2011 \mathrm{JW}_{31}$ & ibr002gaq & 2456188.59889 & $2012 / 09 / 18.09889$ & F606W & 455 & $\mathrm{~N} / \mathrm{A}$ \\
\hline $2011 \mathrm{JW}_{31}$ & ibr002gbq & 2456188.60736 & $2012 / 09 / 18.10736$ & F814W & 640 & $\mathrm{~N} / \mathrm{A}$ \\
\hline $2011 \mathrm{JW}_{31}$ & ibr002gdq & 2456188.61663 & $2012 / 09 / 18.11663$ & F814W & 640 & $\mathrm{~N} / \mathrm{A}$ \\
\hline $2011 \mathrm{JW}_{31}$ & ibr002gfq & 2456188.62510 & $2012 / 09 / 18.12510$ & F606W & 455 & $\mathrm{~N} / \mathrm{A}$ \\
\hline $2011 \mathrm{JY}_{31}$ & ibr011ipq & 2456189.14857 & $2012 / 09 / 18.64857$ & F606W & 455 & $24.64 \pm 0.03$ \\
\hline $2011 \mathrm{JY}_{31}$ & ibr011iqq & 2456189.15704 & $2012 / 09 / 18.65704$ & F814W & 640 & $23.52 \pm 0.03$ \\
\hline $2011 \mathrm{JY}_{31}$ & ibr011isq & 2456189.20856 & $2012 / 09 / 18.70856$ & F814W & 640 & $23.63 \pm 0.04$ \\
\hline $2011 \mathrm{JY}_{31}$ & ibr011iuq & 2456189.21703 & $2012 / 09 / 18.71703$ & F606W & 455 & $24.69 \pm 0.04$ \\
\hline $2011 \mathrm{JY}_{31}$ & ibr012iwq & 2456189.28152 & $2012 / 09 / 18.78152$ & F606W & 455 & $24.66 \pm 0.04$ \\
\hline $2011 \mathrm{JY}_{31}$ & ibr012ixq & 2456189.28999 & 2012/09/18.78999 & F814W & 640 & $23.83 \pm 0.04$ \\
\hline $2011 \mathrm{JY}_{31}$ & ibr012izq & 2456189.34353 & $2012 / 09 / 18.84353$ & F814W & 640 & $23.79 \pm 0.04$ \\
\hline $2011 \mathrm{JY}_{31}$ & ibr012j1q & 2456189.35200 & $2012 / 09 / 18.85200$ & F606W & 455 & $24.61 \pm 0.04$ \\
\hline $2011 \mathrm{HZ}_{102}$ & ibr021dpq & 2456188.21791 & 2012/09/17.71791 & F606W & 455 & $25.68 \pm 0.10$ \\
\hline $2011 \mathrm{HZ}_{102}$ & ibr021dqq & 2456188.22638 & $2012 / 09 / 17.72638$ & F814W & 640 & $24.58 \pm 0.08$ \\
\hline $2011 \mathrm{HZ}_{102}$ & ibr021dsq & 2456188.28014 & $2012 / 09 / 17.78014$ & F814W & 640 & $24.63 \pm 0.09$ \\
\hline $2011 \mathrm{HZ}_{102}$ & ibr021duq & 2456188.28861 & $2012 / 09 / 17.78861$ & F606W & 455 & $25.74 \pm 0.09$ \\
\hline $2011 \mathrm{HZ}_{102}$ & ibr022dwq & 2456188.35075 & $2012 / 09 / 17.85075$ & F606W & 455 & $25.72 \pm 0.09$ \\
\hline $2011 \mathrm{HZ}_{102}$ & ibr022dxq & 2456188.35921 & $2012 / 09 / 17.85921$ & F814W & 640 & $24.79 \pm 0.09$ \\
\hline $2011 \mathrm{HZ}_{102}$ & ibr022dzq & 2456188.39891 & $2012 / 09 / 17.89891$ & F814W & 640 & $24.63 \pm 0.09$ \\
\hline $2011 \mathrm{HZ}_{102}$ & ibr022e1q & 2456188.40738 & $2012 / 09 / 17.90738$ & F606W & 455 & $25.66 \pm 0.08$ \\
\hline $2013 \mathrm{LU}_{35}$ & ibzx01g4 & 2456562.99918 & $2013 / 09 / 27.49918$ & F606W & 455 & $26.41 \pm 0.18$ \\
\hline $2013 \mathrm{LU}_{35}$ & ibzx01g5 & 2456563.00765 & $2013 / 09 / 27.50765$ & F814W & 640 & $25.28 \pm 0.15$ \\
\hline $2013 \mathrm{LU}_{35}$ & ibzx01g7 & 2456563.01692 & $2013 / 09 / 27.51692$ & F814W & 640 & $25.19 \pm 0.12$ \\
\hline $2013 \mathrm{LU}_{35}$ & ibzx01g9 & 2456563.02539 & $2013 / 09 / 27.52539$ & F606W & 455 & $26.40 \pm 0.15$ \\
\hline $2013 \mathrm{LU}_{35}$ & ibzx02jg & 2456563.72922 & $2013 / 09 / 28.22922$ & F606W & 455 & $26.28 \pm 0.13$ \\
\hline $2013 \mathrm{LU}_{35}$ & ibzx02jh & 2456563.73769 & $2013 / 09 / 28.23769$ & F814W & 640 & $25.20 \pm 0.11$ \\
\hline $2013 \mathrm{LU}_{35}$ & ibzx02jj & 2456563.74696 & $2013 / 09 / 28.24696$ & F814W & 640 & $25.24 \pm 0.12$ \\
\hline $2013 \mathrm{LU}_{35}$ & ibzx02jl & 2456563.75542 & $2013 / 09 / 28.25542$ & F606W & 455 & $26.28 \pm 0.13$ \\
\hline
\end{tabular}

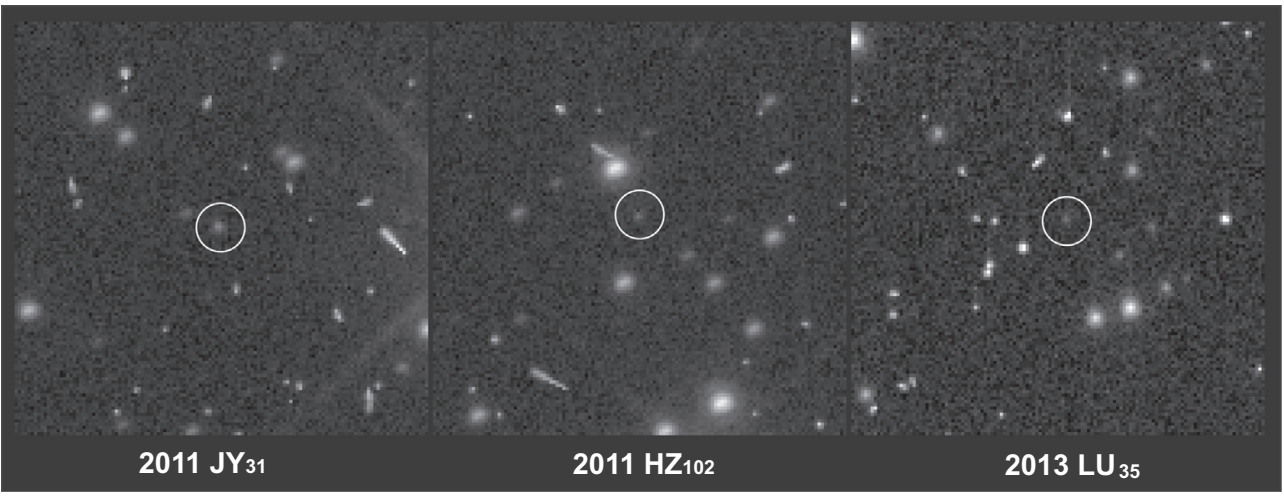

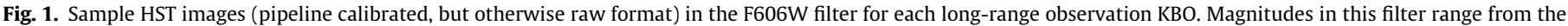
brightest, $2011 \mathrm{JY}_{31}$ at 24.6 to the faintest, $2013 \mathrm{LU}_{35}$ at 26.3.

of sky motion of the KBO. Registration offsets are non-linear due to the parallax from HST's orbit around the Earth. This process led to the recovery of three of the four KBOs observed. One of the targeted KBOs, $2011 \mathrm{JW}_{31}$, was not found. The expected recovery area was unluckily in a cluster of fainter stars not bright enough to be visible in the field pre-selection images. It was also one of the faintest of our first three original sources (Cycle 19: $m_{2011} \mathrm{JY} 31 \sim 24.7, m_{2011} \mathrm{JW} 31 \sim 25.4, m_{2011}$ HZ102 $\sim 25.4$; Cycle 20 : $m_{2013}$ LU35 $\sim 26.0$, with uncertainties as large as a few tenths of a magnitude). As pointed out in Benecchi et al. (2013), KBOs in this size range often exhibit substantial lightcurves, varying up to a full magnitude, on relatively short timescales ( $\sim 8 \mathrm{~h}$ or less). The combination of these factors may have led to the nondetection of this object, or perhaps the ephemeris was not as good as predicted.

The other three objects were detected in the stacked images. Once identified it was possible to identify them in individual images. In a few individual images, the KBOs were close to bright stars or impacted by a cosmic ray or bad pixel. Photometry was carried out using a small aperture on both the KBO and nearby close stars with clean backgrounds. Because KBOs move between frames relative to the background stars, for the few KBO frames with star contamination, the photometry of the star was measured on images when the KBO was not overlapping. On the frames with the overlapping sources, the combined source was measured and the star counts from the other frame were subtracted out to give just the counts for the $\mathrm{KBO}$ on the contaminated frame (with slightly larger uncertainties). In one case cosmic ray contamination in a single pixel was corrected by replacing that pixel with the average of the neighboring pixels, again with slightly larger uncertainties on the resulting magnitude. The background sky was determined using the Goddard Space Flight Center (GSFC) IDL sky routine, part of the IDL Astronomy Library (http://idlastro.gsfc.nasa.gov), in all cases. 
The calibrated fluxes, expressed in the Vega magnitude system (VEGMAG), were calculated from the observed counts using the inverse sensitivity and photometric zeropoint keyword values (PHOTFLAM and PHOTZPT) from the HST image headers (Rajan et al., 2011). We used the synthetic IRAF photometry routine synphot to convert from the STMAG to the VEGAMAG system. F606W and F814W average magnitudes for each HST orbit were then found and the larger of either the combined uncertainties or the scatter in the values ( 2 data points per orbit per filter, 4 per object per filter for the pair of orbits) were used to set the magnitude uncertainties. Each visit also provided a color measurement that was then averaged between the two HST visits for an overall color determination with the same procedure as in the individual orbits used to set the uncertainties. The photometric and color results can be found in Tables 2 and 3.

\subsection{Astrometry}

Our observations confirm that ground-based discovery ephemerides with arcs of $\sim 30$ days are sufficient for recovery in the WFC3 field of view up to a year later. To provide a reference point, for an object such as $2011 \mathrm{JY}_{31}$, which has an orbital period of 292 years, the original arc is significantly improved at each interval where astrometry is measured and as the orbital arc-length grows the positional uncertainty shrinks rapidly as can be demonstrated by modeling (Wasserman et al., 2006) using orbit codes such as Bernstein and Khushalani (2000). The discovery arc, typically $24 \mathrm{~h}$, covers only $0.0009 \%$ of the orbit. We use the Minor Planet Center (MPC) astrometric record of $2011 \mathrm{JY}_{31}$ for illustration (some of the measurements included in the values for Table 1 have yet to be reported to the MPC). This object was discovered on 18 April 2011 and re-observed 6 days, 34 days, 65 days, 1 year and 1.4 years later. With a 6 day arc the total error on the object position is 0.2 arcsec, or 736 arcsec if propagated a year forward. At each progressive interval the immediate error falls to a tenth of an arcsec, while the propagated errors are $42,40,11$ and 0.3 arcsec, respectively. For our HST observations which occurred $\sim 510$ days (1.4 years) from discovery (the final astrometric point previously mentioned) with a ground-based arc of 1 year, the object was recovered within $\sim 10$ HST pixels $(\sim 0.4$ arcsec) of the predicted location. The other recovered objects had similar recovery and recovered properties.

The accuracy of the astrometry also plays a key role in targeting precision since uncertainties in the stellar positions translate to uncertainties in the true position of the KBO at a given time. Relative astrometry from HST for faint targets such as these have a precision of $\sim 1$ pixel or 0.04 arcsec. Absolute astrometry is accurate to 200 mas, limited by the accuracy of astrometric catalogs used to establish the world coordinate system in HST images. For each HST image, astrometry was carried out following the method of Benecchi and Noll (2010). The astrometric results, including the geocentric position of HST at the time of observation, were reported to the Minor Planet Center for improved ephemerides.

Future improved global astrometric catalogs either from ground-based surveys using facilities like the CFHT or in the long term with astrometry from the GAIA mission will allow groundbased and HST astrometry to be redone for improved network and targeting accuracy for fly-by or long range observations by New Horizons. Stellar astrometry from GAIA, expected to be at the level of 24 microarcsec and anticipated to be available within a year or two of GAIA's launch, but well before final corrections would be made for a KBO long-range observation or encounter, will improve our results by $\sim 2$ orders of magnitude (Buie, private communication). Preliminary calculations suggest that a 3-year baseline orbit can be retroactively improved to the equivalent precision of a 34-year baseline (12\% of a typical $\sim 275$ year orbit period for a KBO with $a=42 \mathrm{AU}$ ), making KBO fly-by or long-range observation planning considerably easier.

\section{Binarity, color and size}

Our HST observations allow us the ability to 'pre-classify' potential long range and encounter candidates in order to prioritize the scientific return for the New Horizons spacecraft observations. The New Horizons spacecraft's trajectory through the Pluto system sends it through the region of space populated by the low-inclination, low-eccentricity Cold Classical Kuiper Belt. This is a population that studies show is distinctive both dynamically and photometrically from all other minor planet populations (Elliot et al., 2005; Fraser and Brown, 2012) and may be the most primordial population of objects that can be observed in the Solar System. Oort cloud comets, which reside farther out, are believed to have formed closer to the Sun and then ejected outward, so while they are in cold storage, they are likely to be more processed than the KBOs in the fly-by region. Cold Classical KBOs are thought to have formed near their current location and not to have been substantially scattered during the giant planet migrations (Parker and Kavelaars, 2010; Batygin et al., 2011; Volk and Malhotra, 2011). These KBOs are found to be statistically redder than other populations (Gulbis et al., 2006; Peixinho et al., 2008; Benecchi et al., 2011), to have high albedos (Brucker et al., 2009) and $29_{-6}^{+7} \%$ (Noll et al., 2008) are found to be binary (although we do not know if this binary rate holds for smaller objects, Nesvorný et al., 2011).

To address the question of binary systems, we examined the individual KBO PSFs to look for evidence of binarity. In all cases, the results from HST were negative. No companions are detected with separations of $\sim 0.02 \operatorname{arcsec}(\sim 700 \mathrm{~km}$ at $44 \mathrm{AU})$ and $\Delta m \leqslant 0.5$. Closer undetected companions may yet be detectable with long-range observations from New Horizons where the angular resolution exceeds that of HST (Parker et al., 2013b).

The observed colors of the three new KBOs are shown in Fig. 2 where we compare the newly obtained data with 144 KBO colors

Table 3

Photometry results.

\begin{tabular}{|c|c|c|c|c|c|c|c|c|}
\hline Object & JD (mid-time) & $R(\mathrm{AU})$ & $\Delta(\mathrm{AU})$ & $\alpha\left({ }^{\circ}\right)$ & F606W avg & $\mathrm{F}^{2} 14 \mathrm{~W}_{\mathrm{avg}}$ & F606W-F814W & $\mathrm{V}-\mathrm{I}$ \\
\hline $2011 \mathrm{JY}_{31}$ & 2456189.18280 & - & - & - & $24.67 \pm 0.03$ & $23.57 \pm 0.08$ & $1.09 \pm 0.08$ & $1.37 \pm 0.08$ \\
\hline $2011 \mathrm{JY}_{31}$ & 2456189.31676 & - & - & - & $24.64 \pm 0.04$ & $23.81 \pm 0.03$ & $0.82 \pm 0.05$ & $1.13 \pm 0.05$ \\
\hline $2011 \mathrm{JY}_{31, \mathrm{avg}}$ & 2456189.24978 & 42.71 & 42.50 & 1.32 & $24.65 \pm 0.03$ & $23.69 \pm 0.16$ & $0.96 \pm 0.19$ & $1.25 \pm 0.19$ \\
\hline $2011 \mathrm{HZ}_{102}$ & 2456188.25326 & - & - & - & $25.71 \pm 0.06$ & $24.63 \pm 0.06$ & $1.11 \pm 0.05$ & $1.41 \pm 0.05$ \\
\hline $2011 \mathrm{HZ}_{102}$ & 2456188.37906 & - & - & - & $25.69 \pm 0.06$ & $24.71 \pm 0.11$ & $0.98 \pm 0.12$ & $1.28 \pm 0.12$ \\
\hline $2011 \mathrm{HZ}_{102, \text { avg }}$ & 2456188.31616 & 43.09 & 42.87 & 1.31 & $25.70 \pm 0.02$ & $24.66 \pm 0.07$ & $1.04 \pm 0.15$ & $1.34 \pm 0.15$ \\
\hline $2013 \mathrm{LU}_{35}$ & 2456563.01229 & - & - & - & $26.41 \pm 0.11$ & $25.23 \pm 0.09$ & $1.17 \pm 0.07$ & $1.50 \pm 0.07$ \\
\hline $2013 \mathrm{LU}_{35}$ & 2456563.74233 & - & - & - & $26.28 \pm 0.09$ & $25.22 \pm 0.08$ & $1.06 \pm 0.03$ & $1.39 \pm 0.03$ \\
\hline $2013 \mathrm{LU}_{35, \mathrm{avg}}$ & 2456563.37731 & 44.43 & 44.37 & 1.29 & $26.34 \pm 0.09$ & $25.23 \pm 0.02$ & $1.11 \pm 0.18$ & $1.44 \pm 0.18$ \\
\hline
\end{tabular}


that have been measured in the same HST filters (Benecchi et al., 2009; Fuentes et al., 2010; Fraser and Brown, 2012). The F606WF814W colors (a proxy for $\mathrm{V}-\mathrm{I}$ ) plotted span a wide range from $0.5<$ F606W-F814W $<1.3$. The three newly detected KBOs are at the red end of the range with $\mathrm{F} 606 \mathrm{~W}-\mathrm{F} 814 \mathrm{~W}>0.75$ (including the moderately large $1 \sigma$ uncertainties). The red colors are consistent with the expectation that most of the targets in the search area will be members of the Cold Classical population. All three long-range New Horizons KBO observation candidates observed by HST are significantly fainter than the other KBOs plotted here and are among the faintest to have had their colors measured.

Using the formalism of Bowell et al. (1989) and an assumed geometric albedo of $\rho=0.1$, our long-range targets have diameters that range from 50 to $100 \mathrm{~km}$. These objects are in the approximate size range where sizable amplitude lightcurves ( $>0.2$ magnitudes) might be expected based on results from Trilling and Bernstein (2006) and Benecchi and Sheppard (2013). Absolute variations in magnitude for these objects during a single HST orbit and between pairs of orbits are consistent with the uncertainties in our measurements ( $\sim 0.1$ magnitudes) so at least at our sparsely sampled intervals we do not find these objects to have large amplitude lightcurves indicative of highly elongated shapes.

\section{Science enabled by long range and encounter KBO observations from New Horizons}

Combining our HST results with ground-based detection limits, we expect that the New Horizons spacecraft encounter object will likely be fainter than our current ground-based delectability limit

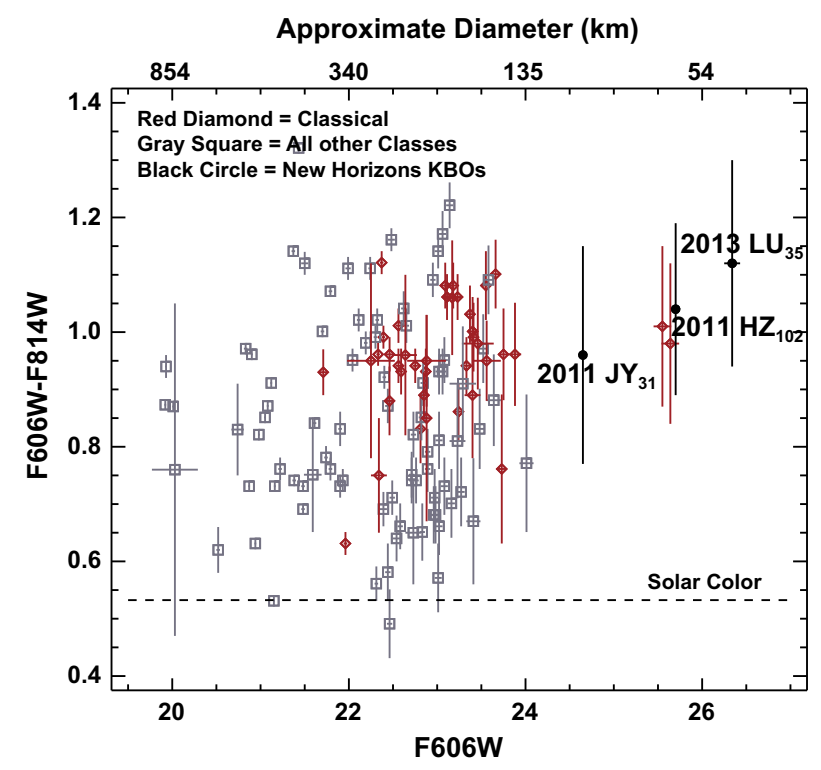

Fig. 2. Comparison of New Horizon long-range candidate objects with the color of other KBOs that have been measured in the same HST filters (from Benecchi et al., 2009; Fuentes et al., 2010; Fraser and Brown, 2012) with respect to their direct F606W magnitudes. Plotted on the top is an estimate of the effective diameters for these objects following the formulation of Bowell et al. (1989) $d=10((6.259-$ $\left.\left.0.4 \cdot H_{\mathrm{F} 606 \mathrm{~W}}-\log \rho\right) / 2\right) \mathrm{km}$ and assuming an albedo, $\rho$; we assume $\rho=0.1$. The F606W-F814W color is similar to ground based Johnson V-Cousins I. Objects plotted as red diamonds are dynamically part of the Cold Classical population, objects in all other classes (scattered, resonant, hot classical and centaur combined) are plotted as gray squares. The color of the Sun in the HST filter set is plotted as a dashed line. The newly measured objects are plotted as black circles and, except for the two Fuentes et al. objects, are fainter by nearly a magnitude or more than the rest of the observed population. These objects are also comparatively in the red portion of the diagram consistent with residing in the Cold Classical region of the Kuiper Belt. (For interpretation of the references to color in this figure legend, the reader is referred to the web version of this article.) of $m_{R}=26.0$ in good seeing $(0.45$ arcsec $)$ over a small fraction of the search area and limit of $m_{E}=25.6$ over $75 \%$ of the search area, giving it a likely diameter of $\leqslant 65 \mathrm{~km}$ (assuming an albedo of $\rho=0.1$; Bowell et al., 1989). A higher albedo, not unexpected for objects in this subpopulation of the Kuiper Belt (Brucker et al., 2009), corresponds to a smaller object. Schlichting et al. (2013), in comparative studies of the observed KBO size distribution with theoretical models, suggest that a diameter of $\sim 60 \mathrm{~km}$ is an important size threshold for studying collisional processing in the Kuiper Belt. They propose that objects above this size are primordial (unmodified by collisional evolution over the age of the Solar System) while smaller objects have been modified by collisions. Therefore, observations of both the size distribution of KBOs in this size regime, as well as high resolution studies of these objects with the New Horizons spacecraft with either long range observations or a close fly-by (Table 1 provides estimated observational resolution for each long-range observation candidate), can provide valuable knowledge about the growth and destruction of large KBOs.

For a likely encounter distance $(10,000-20,000 \mathrm{~km})$, New Horizon's instruments can provide broadband and multicolor images with resolutions of $\sim 100$ and $\sim 400 \mathrm{~m}$, respectively; near-IR spectral maps with $\sim 2 \mathrm{~km}$ resolution sensitive to volatiles such as water ice, methane ice and ammonia; and UV spectra and charged-particle data sensitive to active volatile loss, in addition to concurrent heliocentric plasma and dust-particle measurements (Weaver et al., 2008). Comparison of the characteristics of the Pluto system, thought to be formed through a large collision (Canup, 2011), with studies of the anticipated 'small' KBO to be encountered by New Horizons, will provide a sample of the two different size regimes of the Kuiper Belt population (Stern and Spencer, 2003), which have very different formation and evolutional histories.

\section{Conclusions}

We have reported on the basic photometric characteristics of three KBOs that are observable from the New Horizons spacecraft at long range $(<0.3 \mathrm{AU})$ after the Pluto system fly-by science has been completed. We do not identify any of these objects as binary with HST at the level of 0.02 arcsec separation $(\sim 700 \mathrm{~km})$ and $\Delta m<0.5$. However, the region in which New Horizons will fly is near the center of the Cold Classical Kuiper Belt where nearly $30 \%$ of objects are binary (Noll et al., 2008) and at $<0.3$ AU New Horizons has $\sim 5 \times$ the resolution and sensitivity of HST and could detect companions unresolvable from HST. The three newly discovered objects are all red in color with $0.75<\mathrm{F} 606 \mathrm{~W}-$ F814W $<1.2$ consistent with being members of the Cold Classical population. Distant flybys will enable photometry at phase angles unobservable from the Earth that can eventually be crosscalibrated with the observations at low phase angle reported here. The utility of HST astrometry from our measurements can be improved by future upgrades to absolute astrometry from improved ground (CFHT) or space-based (GAIA) stellar astrometry so that these objects can be targeted accurately with the New Horizons spacecraft for long range observation, with better resolution than any Earth based facility.

\section{Acknowledgments}

This paper is based in part on ground-based data collected at the Subaru Telescope, which is operated by the National Astronomical Observatory of Japan, and on data gathered with the $6.5 \mathrm{~m}$ Magellan Telescopes located at Las Campanas Observatory, Chile. Space-based observations were made with the NASA/ESA Hubble 
Space Telescope, obtained at the Space Telescope Science Institute, which is operated by the Association of Universities for Research in Astronomy, Inc., under NASA contract NAS 5-26555. These observations are associated with programs 12535 and 12887. Support for both programs were provided by NASA through a grant from the Space Telescope Science Institute, which is operated by the Association of Universities for Research in Astronomy, Inc., under NASA contract NAS 5-26555. S. Benecchi was also supported in part through a Carnegie Fellowship at the Department of Terrestrial Magnetism. We thank Dr. Larry Wasserman and an anonymous reviewer for their helpful comments which improved this manuscript.

\section{References}

Batygin, K., Brown, M.E., Fraser, W.C., 2011. Retention of a primordial Cold Classical Kuiper Belt in an instability-driven model of Solar System formation. Astrophys. J. 738, 13. http://dx.doi.org/10.1088/0004-637X/738/1/13.

Belton, M. et al., 2002. New Frontiers in the Solar System: An Integrated Exploration Strategy. National Academies Press, Washington, DC.

Benecchi, S.D., Noll, K.S., 2010. Hubble Space Telescope astrometry of transneptunian objects. Astrophys. J. Suppl. 189 (2), 336-340. http://dx.doi.org/ 10.1088/0067-0049/189/2/336.

Benecchi, S.D., Sheppard, S.S., 2013. Light curves of 32 large transneptunian objects. Astron. J. 145 (5), 124. http://dx.doi.org/10.1088/0004-6256/145/5/124.

Benecchi, S.D., Noll, K.S., Grundy, W.M., Buie, M.W., Stephens, D.C., Levison, H.F., 2009. The correlated colors of transneptunian binaries. Icarus 200, 292. http:/ dx.doi.org/10.1016/j.icarus.2008.10.025.

Benecchi, S.D., Noll, K.S., Stephens, D.C., Grundy, W.M., Rawlins, J., 2011. Optical and infrared colors of transneptunian objects observed with HST. Icarus 213 (2), 693-709.

Bernstein, G.M., Khushalani, B., 2000. Orbit fitting and uncertainties for Kuiper Belt Objects. Astron. J. 120, 3323. http://dx.doi.org/10.1086/316868.

Bowell, E., Hapke, B., Domingue, D., et al., 1989. In: Binzel, R.P., Gehrels, T., Matthews, M.S. (Eds.), Asteroids II. Univ. Arizona Press, Tucson, AZ, pp. 524556.

Brucker, M.J., Grundy, W.M., Stansberry, J.A., Spencer, J.R., Sheppard, S.S., Chiang, E.I., Buie, M.W., 2009. High albedos of low inclination Classical Kuiper Belt Objects. Icarus 201, 1-11. http://dx.doi.org/10.1016/j.icarus.2008.12.040.

Buie et al., 2012. Searching for KBO flyby targets for the New Horizons mission. In: Asteroids, Comets, Meteors 2012, Proceedings of the Conference held May 1620, 2012 in Niigata, Japan. LPI Contribution No. 1667, id.6430.

Canup, R.M., 2011. On a giant impact origin of Charon, Nix, and Hydra. Astron. J. 141, 35. http://dx.doi.org/10.1088/0004-6256/141/2/35.

Cheng, A.F. et al., 2008. Long-range reconnaissance imager on New Horizons. Space Sci. Rev. 140 (1), 189-215. http://dx.doi.org/10.1007/s11214-007-9271-6.

Elliot, J.L. et al., 2005. The deep ecliptic survey: A search for Kuiper Belt Objects and centaurs. II. Dynamical classification, the Kuiper Belt plane, and the core population. Astron. J. 129 (2), 1117-1162. http://dx.doi.org/10.1086/427395.

Fraser, W.C., Brown, M.E., 2012. The Hubble Wide Field Camera 3 test of surfaces in the outer Solar System: The compositional classes of the Kuiper Belt. Astrophys. J. 749 (1), 33. http://dx.doi.org/10.1088/0004-637X/749/1/33.
Fraser, W.C., Brown, M.E., Morbidelli, A., Parker, A., Batygin, K., 2014. The Absolute Magnitude Distribution of Kuiper Belt Objects. The Astrophysical Journal 782 (2), 100. http://dx.doi.org/10.1088/0004-637X/782/2/100.

Fuentes, C.I., Holman, M.J., Trilling, D.E., Protopapas, P., 2010. Trans-neptunian objects with Hubble Space Telescope ACS/WFC. Astrophys. J. 722 (2), 12901302. http://dx.doi.org/10.1088/0004-637X/722/2/1290.

Gulbis, A.S., Elliot, J.L., Kane, J.F., 2006. The color of the Kuiper Belt core. Icarus 183 $168-178$.

Heyer, I. et al., 2004. WFPC2 Instrument Handbook: Version 9.0. STScI, Baltimore.

Jewitt, D., Luu, J., 1993. Discovery of the candidate Kuiper Belt Object 1992 QB1. Nature 362, 730-732 (ISSN 0028-0836).

Nesvorný, D., Vokrouhlický, D., Bottke, W.F., Noll, K., Levison, H.F., 2011. Observed binary fraction sets limits on the extent of collisional grinding in the Kuiper Belt. Astron. J. 141, 159. http://dx.doi.org/10.1088/0004-6256/141/5/159.

Noll, K.S., Grundy, W.M., Stephens, D.C., Levison, H.F., Kern, S.D., 2008. Evidence for two populations of classical transneptunian objects: The strong inclination dependence of classical binaries. Icarus 194, 758. http://dx.doi.org/10.1016/ j.icarus.2007.10.022.

Parker, A.H., Kavelaars, J.J., 2010. Destruction of binary minor planets during Neptune scattering. Astrophys. J. 722, L204. http://dx.doi.org/10.1088/20418205/722/2/L204

Parker, A.H. et al., 2013a. 2011 HM102: Discovery of a high-inclination L5 Neptune Trojan in the search for a post-Pluto New Horizons target. Astron. J. 145 (4), 96. http://dx.doi.org/10.1088/0004-6256/145/4/96.

Parker, A.H. et al., 2013b. Long-range Kuiper Belt Object observations: Prospects and scientific value. In: New Horizons Science Conference - The Pluto System on the Eve of Exploration by New Horizons: Perspectives and Predictions. APL, 22-26 July 2013.

Peixinho, N., Lacerda, P., Jewitt, D.C., 2008. Color-inclination relation of the Classical Kuiper Belt Objects. Astron. J. 136, 1837. http://dx.doi.org/10.1088/0004-6256/ $136 / 5 / 1837$.

Petit, J.M. et al., 2011. The Canada-France ecliptic plane survey-Full data release: The orbital structure of the Kuiper Belt. Astron. J. 142, 131. http://dx.doi.org/ 10.1088/0004-6256/142/4/131.

Rajan, A. et al., 2011. WFC3 Data Handbook, Version 2.1. STScI, Baltimore, <http:// www.stsci.edu/hst/wfc3/documents/handbooks/currentDHB/wfc3 dhb.pdf>.

Schlichting, H.E., Fuentes, C.I., Trilling, D.E., 2013. Initial planetesimal sizes and the size distribution of small Kuiper Belt Objects. Astron. J. 146 (2), 36. http:// dx.doi.org/10.1088/0004-6256/146/2/36.

Spencer, J.R., Buie, M.W., Young, L.A., Guo, Y., Stern, S.A., 2003. Finding KBO Flyby Targets for New Horizons. Earth, Moon and Planets 92, 483-493. http:/ dx.doi.org/10.1023/B:MOON.0000031963.58573.97.

Spencer, J.R. et al., 2013. The search for KBO flyby targets for New Horizon. In: New Horizons Science Conference - The Pluto System on the Eve of Exploration by New Horizons: Perspectives and Predictions. APL, 22-26 July 2013.

Stern, S.A., 2008. The New Horizons Pluto Kuiper Belt mission: An overview with historical context. Space Sci. Rev, 140, 3-21.

Stern, S.A., Spencer, J.R., 2003. New Horizons: The first reconnaissance mission to bodies in the Kuiper Belt. Earth 92, 477-482.

Trilling, D.E., Bernstein, G.M., 2006. Light curves of 20-100 km Kuiper Belt Objects using the Hubble Space Telescope. Astron. J. 131, 1149. http://dx.doi.org/ $10.1086 / 499228$.

Volk, K., Malhotra, R., 2011. Inclination mixing in the Classical Kuiper Belt Astrophys. J. 736, 11. http://dx.doi.org/10.1088/0004-637X/736/1/11.

Wasserman, L.H. et al., 2006. Statistical ranging as a tool for short-arc KBO recovery and classification. Am. Astron. Soc. 38, 564.

Weaver, H.A., Gibson, W.C., Tapley, M.B., Young, L.A., Stern, S.A., 2008. Overview of the New Horizons science payload. Space Sci. Rev. 140, 75-91. 